\title{
Correction of the allosteric site of E. coli D-3-phosphoglycerate dehydrogenase
}

\author{
D Schuller ${ }^{1}$, T Lukk $^{2}$ \\ ${ }^{1}$ MacCHESS, Cornell Univ, Ithaca, NY, ${ }^{2}$ Tallinn University of Technology, Tallinn, Estonia \\ djs63@cornell.edu
}

D-3-phosphoglycerate dehydrogenase(PGDH), an enzyme in the serine biosynthesis pathway, is known as an example of "Vmax" allosteric regulation and as the archetype structure for the ACT conserved motif. The first structure of E. coli PGDH, published in 1995 by Schuller, et al., presented a tetramer with all of its allosteric sites filled with the inhibitor serine. With better data and new structures, we show here that the location of the serine was correct but its orientation was not. This error in the original structure was propagated to several other published structures. In 2005 a tetragonal structure was published by Thompson, et al. and touted as the uninhibited "active form" of the enzyme. We have duplicated this form with fresh data and structures, and have examined the deposited structure, and state here that this form consistently has one of the four serine sites in the tetramer occupied, which kinetic studies indicate should result in $\sim 50 \%$ inhibition.

Acta Cryst. (2020). A76, a114 